Supporting Information for:

\title{
Hyaluronidase Functionalized Silica Nanocarrier for Enhanced Chemo-Immunotherapy through Inducing Immunogenic Cell Death
}

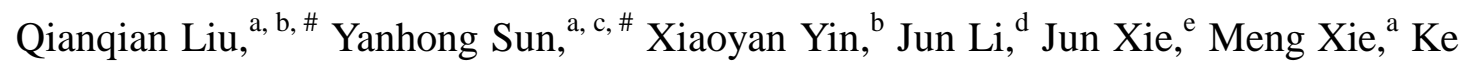
Wang, Shidi Wu, ${ }^{\text {a }}$ Yuce Li, ${ }^{\text {b }}$ Mubashir Hussain, ${ }^{b}$ Biling Jiang, ${ }^{a}$ Yijing Liu, ${ }^{b}$ Changzheng Huang, ${ }^{\mathrm{a}, *}$ Juan Tao, ${ }^{\mathrm{a}, *}$ and Jintao Zhu ${ }^{\mathrm{b}, *}$

${ }^{a}$ Department of Dermatology, Union Hospital, Tongji Medical College, Huazhong University of Science and Technology (HUST), Wuhan 430022, China

b Key Laboratory of Material Chemistry for Energy Conversion and Storage of Ministry of Education, School of Chemistry and Chemical Engineering, HUST, Wuhan 430074, China

c Department of Dermatology, Second Affiliated Hospital, School of Medicine, Zhejiang University, Hangzhou 310009, China

d Department of Dermatology, The Central Hospital of Wuhan, Tongji Medical College, HUST, Wuhan 430022, China

${ }^{\text {e }}$ Department of Dermatology, Zhongnan hospital, Wuhan University, Wuhan 430071, China

\# These authors contributed equally to this work

*Corresponding authors, E-mail: hcz0501@126.com (C. Huang);

tjhappy@126.com (J. Tao);

jtzhu@mail.hust.edu.cn (J. Zhu) 


\section{Supporting figures:}
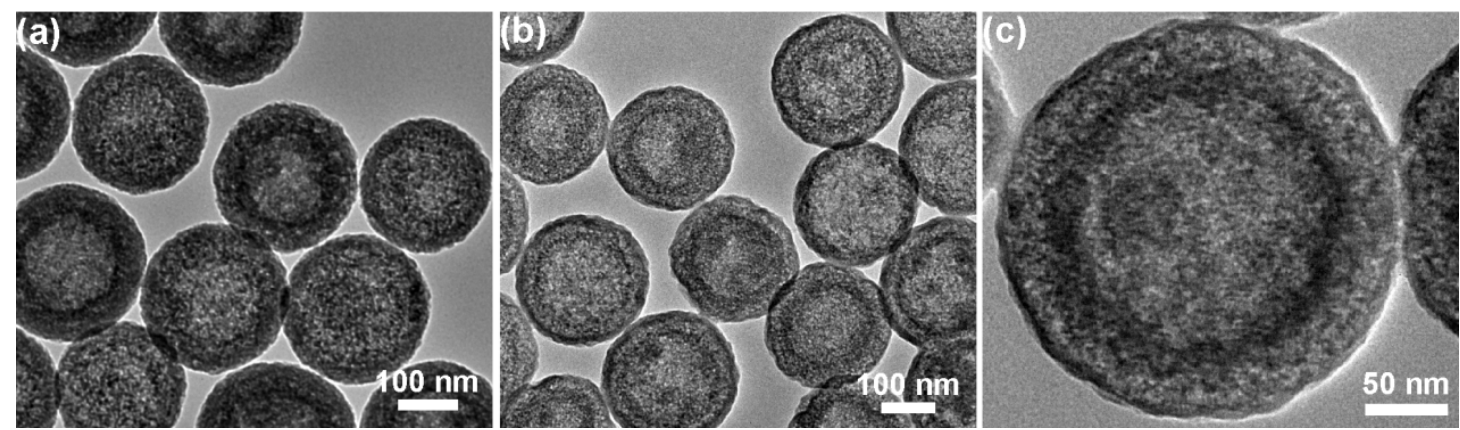

Figure S1. TEM images of (a) HMSPs, HMSPHs with (b) low and (c) high magnification. 


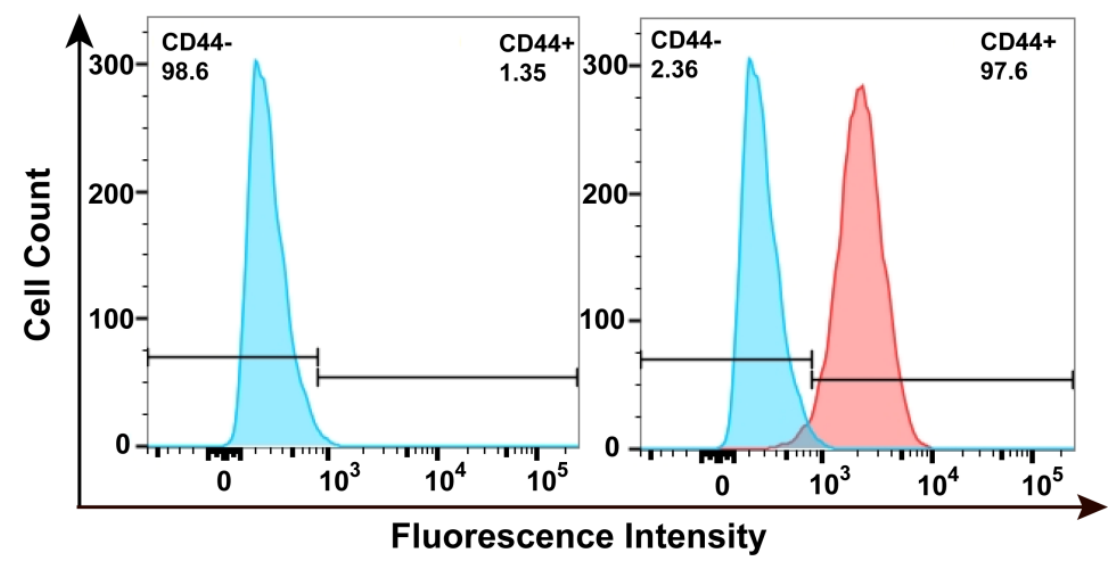

Figure S2. The CD44 expression (red signal) on the surface of B16/F10 by flow cytometry analysis (the blue signal represents blank). 


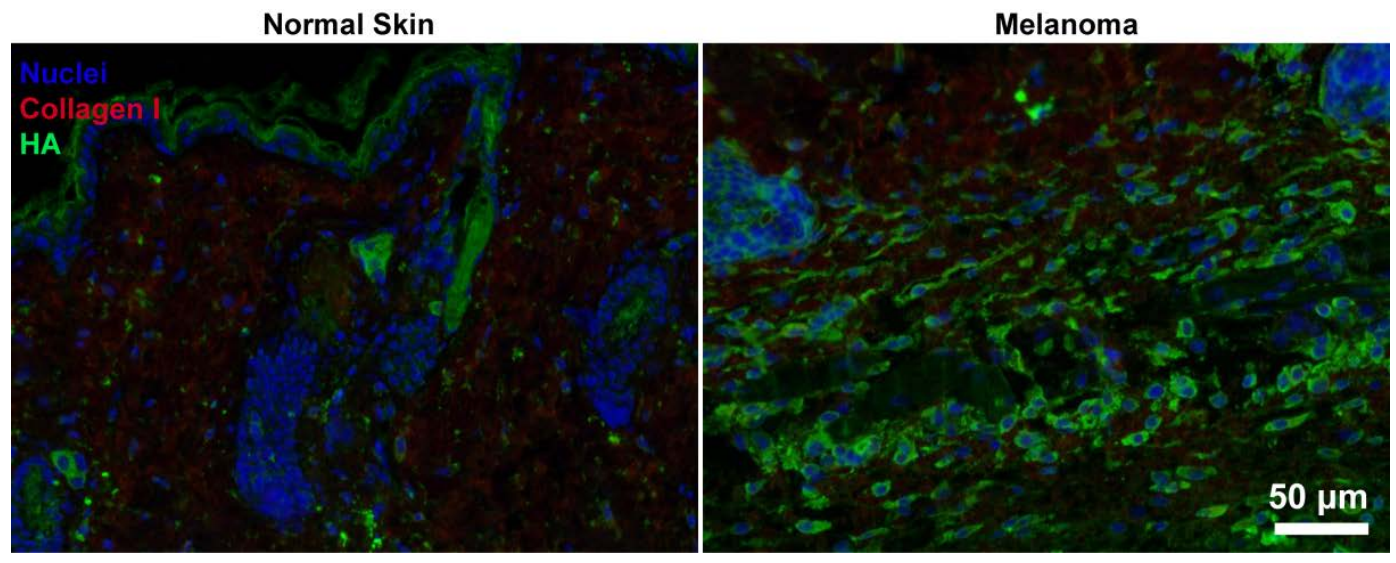

Figure S3. CLSM images of the expression of hyaluronic acid (HA) in normal skin and melanoma of mice. The scale bar in the last image can be applied to the other image. 

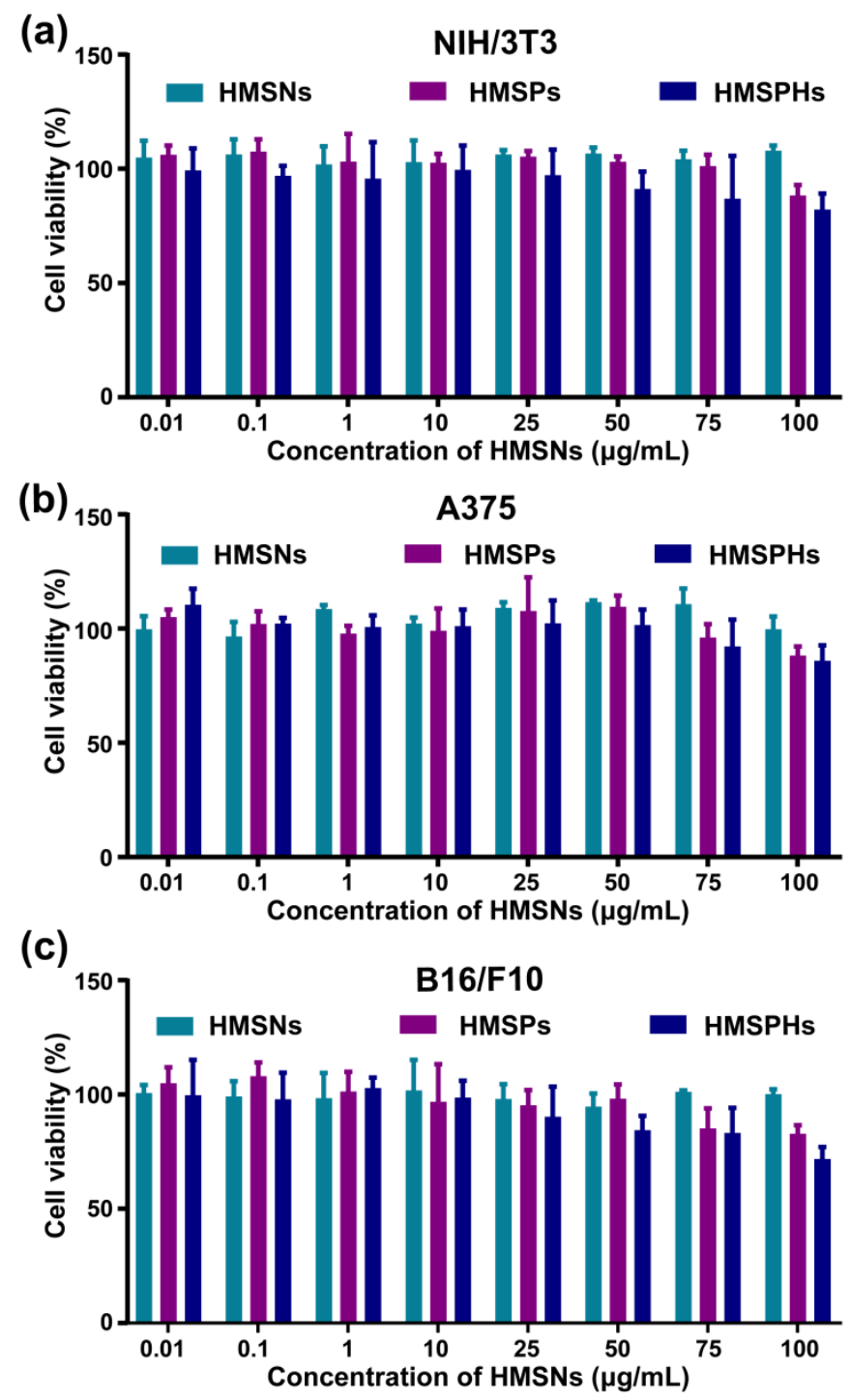

Figure S4. Cell viability of (a) NIH/3T3, (b) A375 and (c) B16/F10 after treatment with HMSNs, HMSPs and HMSPHs for 24 h, respectively. All experiments were performed in triplicate. 


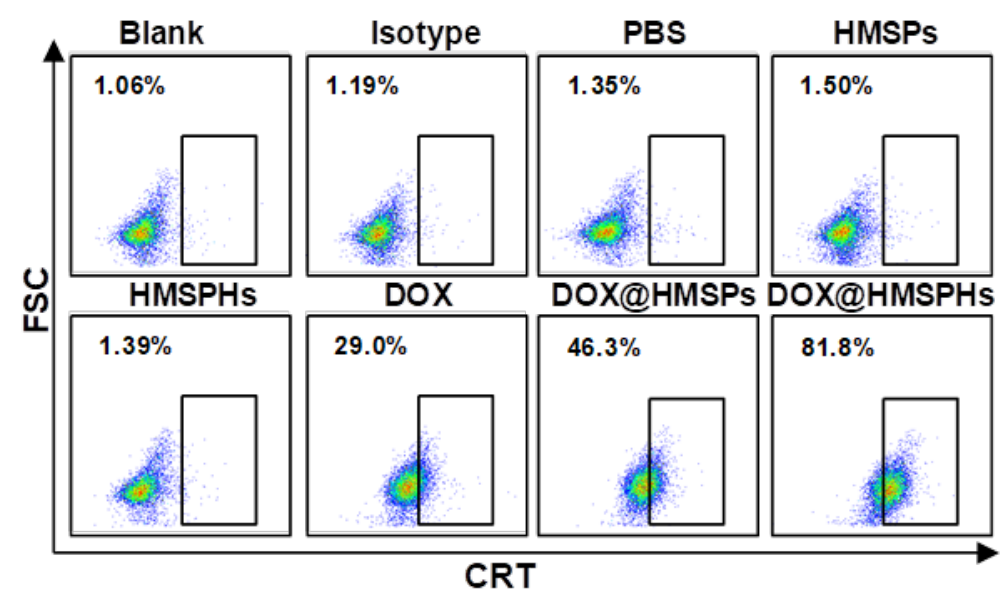

Figure S5. Scatter plots of CRT positive B16/F10 cells after co-incubation with different drug formulations. 


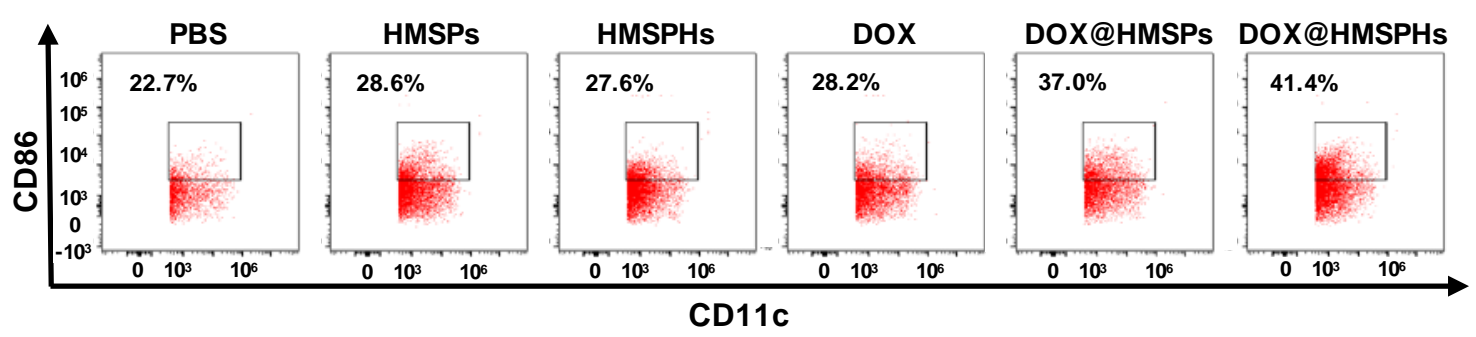

Figure S6. Scatter plots of $\mathrm{CD}^{+} 6^{+}$cells in CD11c ${ }^{+}$cells in TDLNs $(n=3)$. 


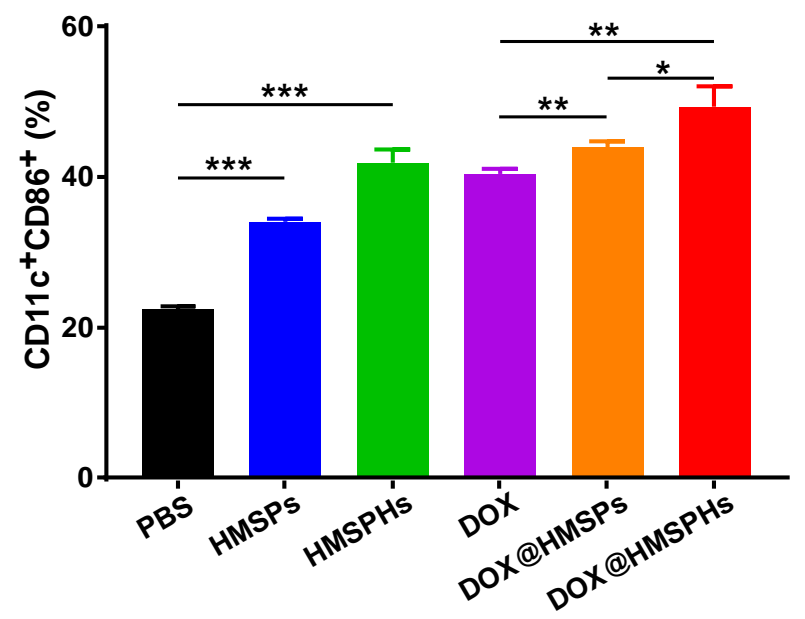

Figure S7. The proportion of $\mathrm{CD}^{+} 6^{+}$cells in $\mathrm{CD} 11 \mathrm{c}^{+}$BMDCs after co-incubation with the different drug formulations-treated B16/F10 cells for 24 h. All experiments were performed in triplicate. 


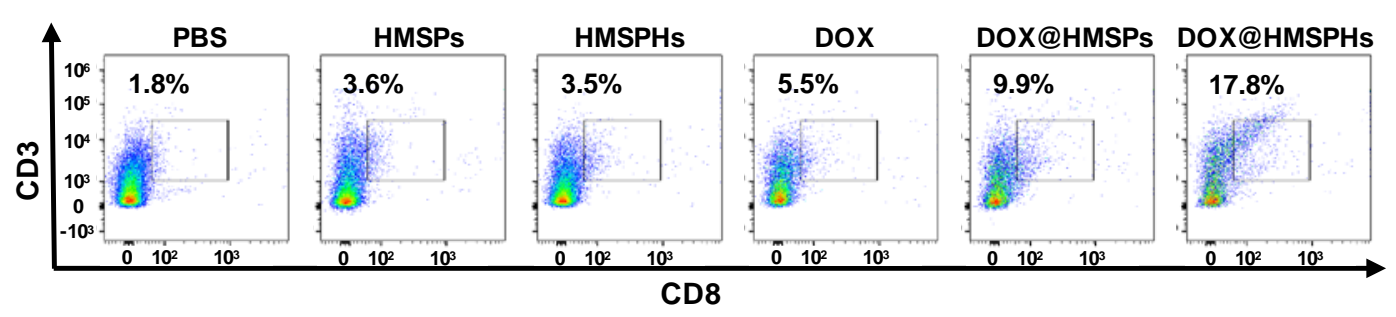

Figure S8. Scatter plots of $\mathrm{CD}^{+} \mathrm{CD}^{+} \mathrm{T}$ lymphocytes in tumor issues $(n=3)$. 


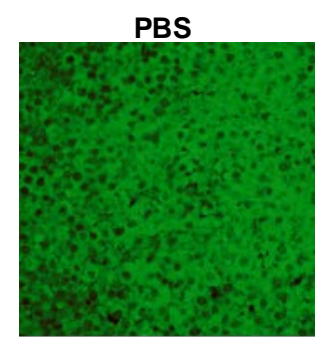

HMSPs

HMSPHs

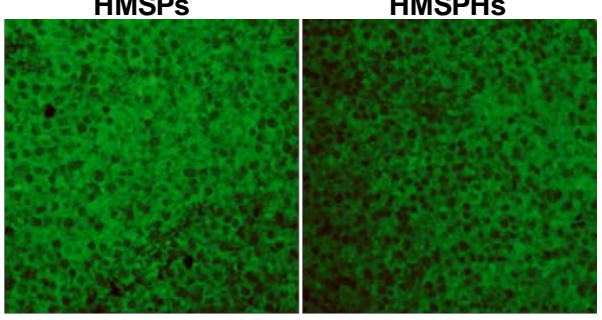

DOX

DOX@HMSPs

DOX@HMSPHs
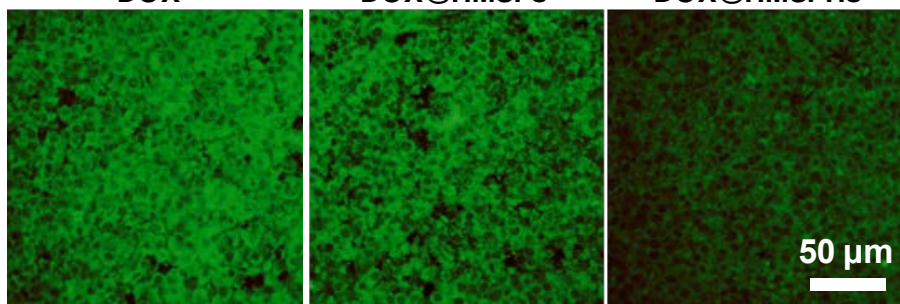

Figure S9. HA staining images of tumors for different groups were shown. The scale bar in the last image can be applied to the other images. 


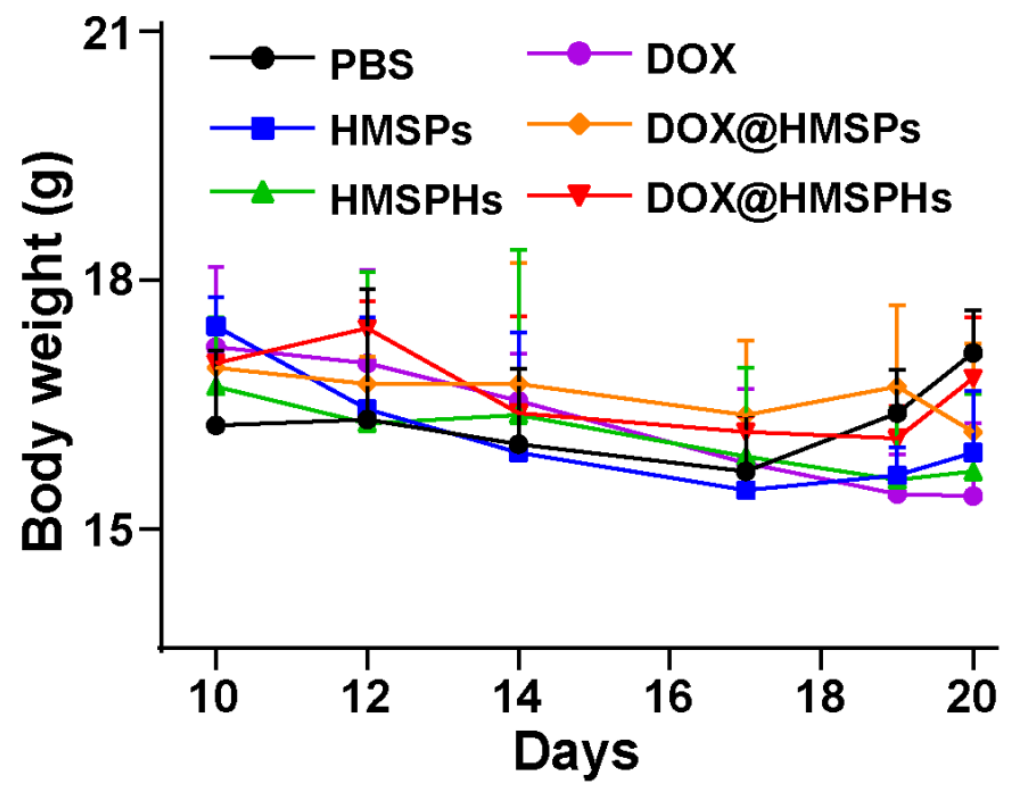

Figure S10. The body weight of the mice from different groups $(n=5)$. 

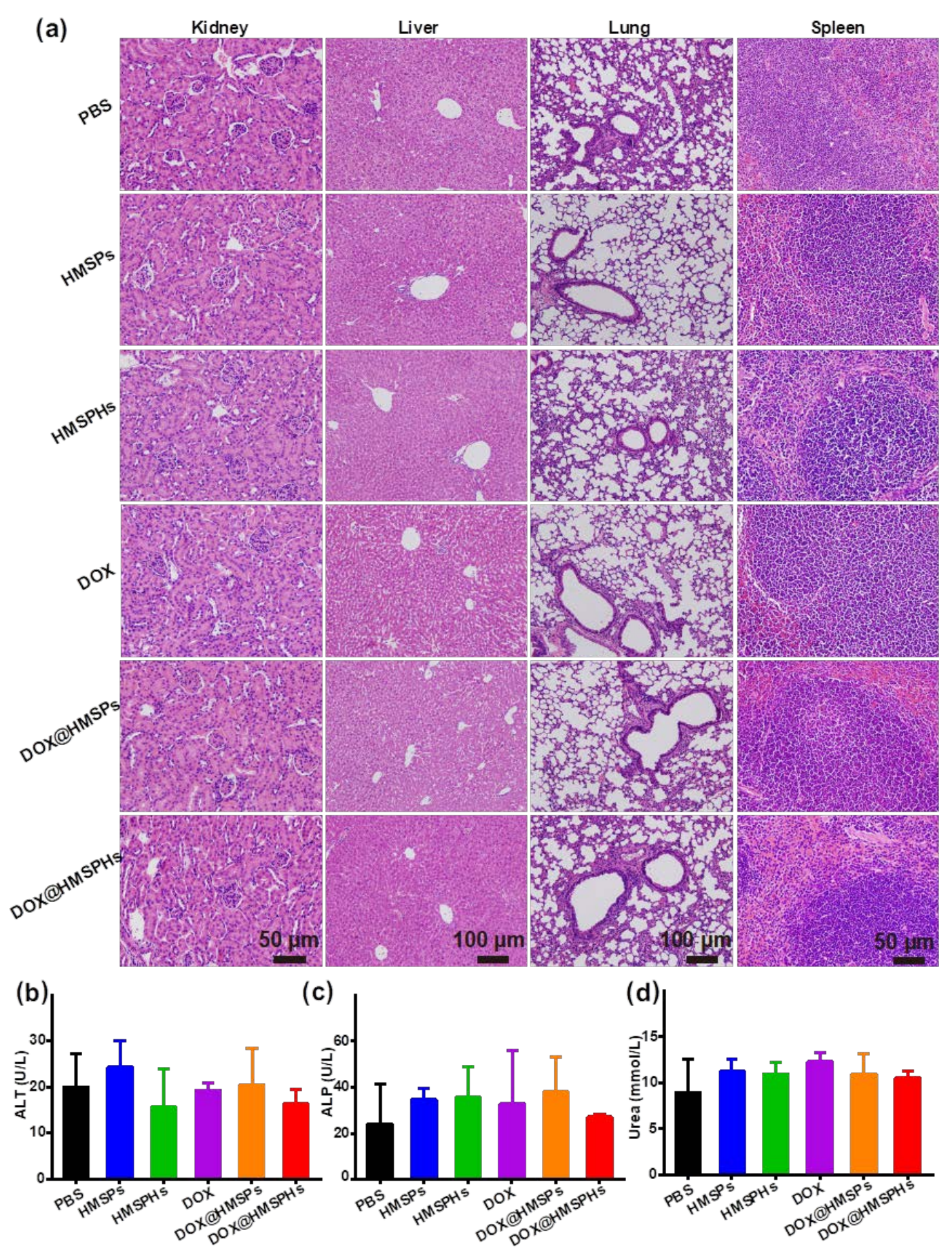

Figure S11. Toxicity tests in vivo after intratumor injection of different drug formulations in mice. (a) H\&E staining images of kidney, liver, lung and spleen of different groups were shown. The levels of (b) ALT, (c) ALP and (d) Urea in mice serum of different groups $(n=3)$. 


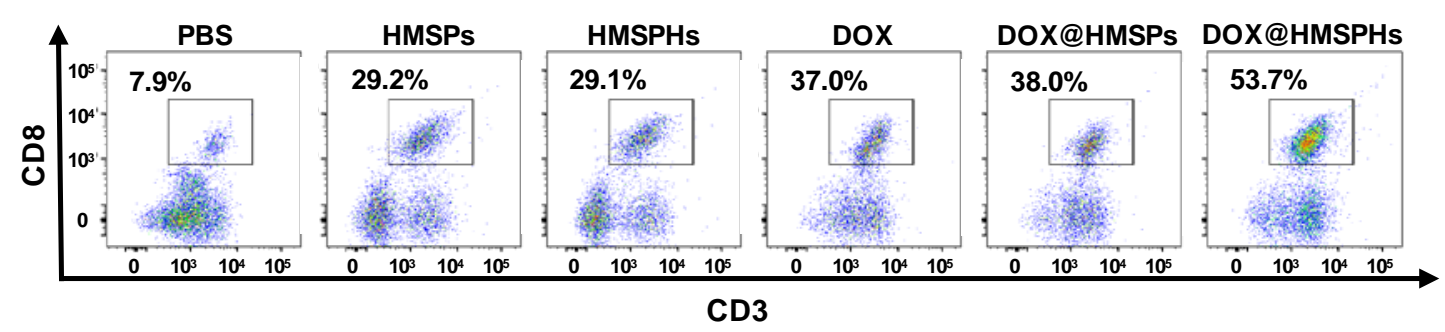

Figure S12. Scatter plots of $\mathrm{CD}^{+} \mathrm{CD}^{+} \mathrm{T}$ lymphocytes cells in total lymphocytes in re-inoculation tumors $(n=3)$. 